\title{
Astronomical Determination of Longitudes and Azimuths
}

$\mathrm{H}$

ISTORICALLY the relations between geodesy and astronomy have always been of the most intimate character, and the contributions of Bessel, Struve and Gill, famous as astronomers, to measured ares and the adjustment of observations still play a fundamental part in the determination of the figure of the earth. It was therefore appropriate, the more so since astronomy is becoming increasingly concerned in its objects and methods with physics, that the Royal Astronomical Society should have recalled its association with geodesy by inviting Prof. N. E. Nörlund, president at the recent London meeting of the International Council of Scientific Unions, to deliver the annual George Darwin Lecture before the Royal Astronomical Society on May 14.

Prof. Nörlund took as his subject the astronomical determination of longitudes and azimuths. He derived a general expression for the error in a longitude determination as a function of the zenith distances of the two groups of stars observed with the transit, and showed that a corresponding expression held for the azimuth determination provided the polar distances of the groups were substituted for the zenith distances. Graphical representation of this expression showed that for the maximum attainable accuracy a considerable freedom in the choice of stars was permissible, and Prof. Nörlund showed how this freedom might be utilized to minimize the unavoidable systematic errors due to level, the positions of the stars and personal equation. This discussion in its turn naturally led to a discussion of the separation of the 'Laplace points', where the geodetic triangulation is adjusted by such astronomical observations.

Prof. Nörland concluded that with stars chosen in accordance with the foregoing considerations and with the methods of observation used in Denmark, a separation of as much as $250 \mathrm{~km}$. was allowable. This separation is determined by the necessity of keeping the labour of observation and adjustment to a minimum and if the occasion had permitted, many fellows of the Society would have been interested in hearing Prof. Nörlund's reactions to the alternative procedure of occupying more 'Laplace points' with the simpler observational procedure made possible by the prismatic astrolabe, or an astrolable attachment to a theodolite.

The lecture was characterized by a number of asides on the technique of observation, and some of the results thereof. None excited greater interest than the concluding remarks on the longitude of western Greenland. An important piece of evidence for Wegener's hypothesis of continental drift has been the fact that longitude determinations in Greenland between 1823 and 1870 , and between 1870 and 1907 showed evidence of a westerly movement of the area amounting to 9 metres per annum in the first period and 32 metres per annum in the second. Under Prof. Nörlund's direction, the Danish Geodetic Survey has occupied stations in western Greenland at two epochs separated by seven years, and in that period there has been no change of longitude. There can therefore be little doubt that Wegener's suspected movement is simply the result of errors in the early determinations of longitude.

\section{Nitrogen Transformations in the Soil}

$\mathrm{I}^{\mathrm{N}}$ his presidential address to the National Academy of Sciences, India, on January 15, Prof. N. R. Dhar gave a very comprehensive account of his work on nitrogen transformations in the soil, various aspects of which have recently been described in NATURe $(137,462,629,1000 ; 138,648,1060 ; 1936)$. Further evidence was provided of increases both in soil nitrogen content and in crop yield following the application of molasses, and striking results were reported in the reclamation of alkali land by molasses on the practical scale. Other carbohydrate-rich materials, and the sodium salts of fatty acids, were also shown to promote nitrogen fixation. Cow dung had a double action, not only supplying extra nitrogen but also leading to nitrogen fixation as well. With more highly nitrogenous materials, however, such as oil cake or sulphate of ammonia, a loss of nitrogen from the soil took place, especially if it was exposed to sunlight. This loss was reduced when leaves or cow-dung were also added; while molasses converted the loss into a gain, nitrogen fixation occurring in spite of the presence of much available nitrogen.

Many experiments were described on the influence of sunlight on ammonification, nitrification, nitrogen fixation and the loss of nitrogen from soils. Prof. Dhar has shown both in the laboratory and in the field that exposure to sunlight accelerates these processes, but it is necessary to point out that the heating effect of the long wave-lengths was present as well as the possibly photochemical effect of the short wave-lengths. Soils exposed to full sunlight were compared with soils that were either shaded or kept in a dark room. The higher temperature of the exposed soils would be expected to eause, on the average, greater microbiological activity, and although the author observed that the temperatures were often above the optima for bacteria in pure culture, it is unsafe to argue from this to mixed soil flora which were subject to the fluctuations between day and night temperature.

This point is raised because, while Prof. Dhar's results are undoubtedly of great interest, the a priori objection to the occurrence of an important amount of photochemical activity in soils in the field, based on their opacity to light, is such a real one that experiments on the subject require especially stringent examination. 